\title{
Cognitive Profile of Idiopathic Normal Pressure Hydrocephalus
}

\author{
Makoto Saito $^{\mathrm{a}}$ Yoshiyuki Nishio $^{\mathrm{a}}$ Shigenori Kanno $^{\mathrm{a}}$ \\ Makoto Uchiyama $^{a}$ Akiko Hayashi ${ }^{a}$ Masahito Takagi ${ }^{a}$ \\ Hirokazu Kikuchi $^{a}$ Hiroshi Yamasaki $^{a}$ Tatsuo Shimomura $^{b}$ \\ Osamu lizuka ${ }^{a}$ Etsuro Mori ${ }^{a}$ \\ a Department of Behavioral Neurology and Cognitive Neuroscience, Tohoku University \\ Graduate School of Medicine, Sendai, and ${ }^{b}$ Department of Rehabilitation Medicine, Akita \\ Prefectural Center of Rehabilitation and Psychiatric Medicine, Daisen, Japan
}

\section{Key Words}

Executive dysfunction - Neuropsychology of dementia - Subcortical dementia •

Visual perception $\cdot$ Visuospatial abilities

\begin{abstract}
Background/Aims: Frontal lobe dysfunction is believed to be a primary cognitive symptom in idiopathic normal pressure hydrocephalus (iNPH); however, the neuropsychology of this disorder remains to be fully investigated. The objective of this study was to delineate a comprehensive profile of cognitive dysfunction in iNPH and evaluate the effects of cerebrospinal fluid (CSF) shunt surgery on cognitive dysfunction. Methods: A total of 32 iNPH patients underwent neuropsychological testing of memory, attention, language, executive function, and visuoperceptual and visuospatial abilities. Of these 32 patients, 26 were reevaluated approximately 1 year following CSF shunt surgery. The same battery of tests was performed on 32 patients with Alzheimer's disease (AD) and 30 healthy elderly controls. Results: The iNPH patients displayed baseline deficits in attention, executive function, memory, and visuoperceptual and visuospatial functions. Impairments of attention, executive function, and visuoperceptual and visuospatial abilities in iNPH patients were more severe than in those with $A D$, whereas the degree of memory impairment was comparable to that in $A D$ patients. A significant improvement in executive function was observed following shunt surgery. Conclusion: Patients with iNPH are impaired in various aspects of cognition involving both 'frontal' executive functions and 'posterior cortical' functions. Shunt treatment can ameliorate executive dysfunction.
\end{abstract}




\section{Introduction}

Idiopathic normal pressure hydrocephalus (iNPH) is a neurological disorder that preferentially occurs in the 6th-7th decades of life [1]. Although iNPH was thought to be a rare disorder, recent epidemiologic studies report its prevalence in the elderly to be $0.1-2.9 \%[2,3]$. Therefore, it is becoming increasingly important to make an early and accurate iNPH diagnosis. iNPH is classically defined as a condition presenting with gait disturbance, cognitive dysfunction and urinary incontinence. Cerebrospinal fluid (CSF) shunt surgery has been reported to improve these symptoms [4,5]. The classic definition of iNPH does not provide any tools for the preoperative differentiation of iNPH from other neurological diseases presenting with similar symptoms. In an effort to address this issue, several guidelines and diagnostic criteria for the preoperative diagnosis of iNPH have been proposed recently [6, 7]. According to these guidelines, the preoperative diagnosis of iNPH is problematic when patients do not present with gait disturbance; however, cognitive dysfunction can be a dominant symptom at an early stage of iNPH $[3,8,9]$. In such cases, detailed characterization of cognitive dysfunction is essential for differentiating this disorder from neurodegenerative dementia.

Many previous studies on cognitive dysfunction in iNPH have focused on attention, executive function and memory [10-12], whereas fewer studies have focused on 'posterior cortical' functions, such as visuoperceptual and visuospatial functions. Given that pathological changes in iNPH are not localized to the frontal lobe but also affect posterior brain regions [13], a more comprehensive characterization of cognitive dysfunction in iNPH patients is necessary. In addition, information regarding the effects of surgical intervention on cognitive dysfunction is scarce. In this context, we characterized cognitive dysfunction in iNPH patients by comparing it to the dysfunction observed in patients with Alzheimer's disease (AD). In addition, we identified the effects of CSF shunt surgery on cognitive dysfunction in patients with iNPH.

\section{Methods}

The Ethical Committee of the Tohoku University Graduate School of Medicine approved all procedures in this study. Written informed consent was obtained from all participants after the study procedure had been fully explained.

\section{Diagnosis of $i N P H$}

To date, there are no accepted criteria by which a reliable diagnosis of preoperative iNPH can be made $[6,7]$. To exclude the possibility of misdiagnosis or comorbidity, we employed the classic diagnostic criterion of iNPH by recruiting only patients who significantly improved (as defined below) after shunt surgery [5].

According to the guidelines of the Japanese Society of Normal Pressure Hydrocephalus, patients were invited for further evaluation if they presented with at least 1 of 3 symptoms (gait disturbance, cognitive dysfunction or urinary incontinence) and ventricular enlargement with a narrowing of the high convexity/midline subarachnoid spaces on magnetic resonance imaging (MRI) [6,9]. Patients suspected of having other diseases, such as AD or Parkinson's disease, were excluded on the basis of neurological examination, neuropsychological testing, laboratory investigations, brain MRI, single photon emission computed tomography and lumbar CSF tap test. The remaining patients received shunt surgery irrespective of their CSF tap test result $[6,9]$. Clinical symptoms before and after CSF shunt surgery were rated using the iNPH Grading Scale (iNPHGS) [14]. On this scale, each of the 3 symptoms is scored from 1 to 4 , and the total score varies from 0 (normal) to 12 (severe). We de- 
Table 1. Demographic profiles of the iNPH, AD and NC groups

\begin{tabular}{lllll}
\hline & iNPH & AD & NC & p value \\
\hline Subjects, n & 32 & 32 & 30 & $0.960\left(\chi^{2}\right.$ test $)$ \\
$\quad$ Females/males & $16 / 16$ & $17 / 15$ & $15 / 15$ & \\
Age, years & $76.3 \pm 4.6$ & $76.0 \pm 5.8$ & $76.8 \pm 5.7$ & $0.860^{\mathrm{a}}$ \\
Education, years & $10.1 \pm 3.6$ & $9.9 \pm 2.5$ & $10.5 \pm 2.8$ & $0.762^{\mathrm{a}}$ \\
CSF shunt operation (VP/LP) & $22 / 10$ & & & \\
\hline
\end{tabular}

Means \pm SD except for number of subjects. ${ }^{a} p$ values are based on one-way analysis of variance. $\mathrm{CSF}=$ Cerebrospinal fluid; VP = ventriculo-peritoneal; $\mathrm{LP}=$ lumbo-peritoneal.

fined a reduction $\geq 1$ point on the total iNPHGS score after surgery compared to the baseline score as a significant improvement.

\section{Subjects}

Patients with iNPH were prospectively recruited from patients admitted to the Department of Behavioral Neurology and Cognitive Neuroscience at the Tohoku University Hospital and the Department of Rehabilitation Medicine at the Akita Prefectural Center of Rehabilitation and Psychiatric Medicine from May 2006 to April 2009. Patients who were unable to complete the neuropsychological tests for clinical reasons, including refusal of the examination, delirium and severe apathy, were excluded from the study. Consequently, 32 patients with iNPH who showed significant improvement after CSF shunt surgery were included in this study. The mean \pm SD iNPHGS scores before and after CSF shunt surgery were $2.5 \pm$ 0.7 and $2.1 \pm 0.8$ (Wilcoxon test, $\mathrm{p}=0.002)$ in cognition, $2.4 \pm 0.7$ and $1.7 \pm 0.9(\mathrm{p}<0.001)$ in gait, $2.0 \pm 1.0$ and $0.9 \pm 1.0(\mathrm{p}<0.001)$ in urination, and $6.9 \pm 1.7$ and $4.7 \pm 1.9(\mathrm{p}<$ $0.001)$ in total. The mean \pm SD interval between the tests and shunt surgery was $1.7 \pm 1.5$ (range, 0-6) months. As disease controls, 32 patients with AD, matched for age, gender, duration of education and degree of cognitive dysfunction assessed by the Mini-Mental State Examination (MMSE), were selected from the same patient pools described above. Diagnosis was made according to the criteria for probable AD of the National Institute of Neurological and Communicative Diseases and Stroke/Alzheimer's Disease and Related Disorders Association [15]. To obtain normative data, 30 healthy elderly controls (normal control group, NC) who were equivalent in age, gender and education were also recruited. The demographic and clinical data of the study participants are summarized in table 1.

A total of 26 (13 women/13 men) of the initial 32 iNPH patients were reevaluated approximately 1 year after the CSF shunt surgery. The other 6 patients withdrew because of refusal, complications or death. Postoperative follow-up (mean \pm SD) lasted $12.6 \pm 1.2$ (range, 11-16) months. Patient age at baseline and duration of education (means \pm SD) were $75.7 \pm 4.5$ (range, 65-84) and $10.0 \pm 3.1$ years, respectively.

\section{Neuropsychological Assessments}

The following neuropsychological tests were administered to evaluate various domains of cognition:

(1) MMSE [16] for general cognitive function.

(2) Digit span and spatial span tests for attention; summed scores of forward and backward spans were used for analysis.

(3) Word fluency [17], Trail Making Test-A (TMT-A) [17] and Frontal Assessment Battery $(\mathrm{FAB})[18]$ for executive function. In the word fluency test, the 1-min free recall of words 
beginning with 'Fu', 'A', 'Ni' (phoneme) and of animal names (category) was tested. In the TMT-A test, the number of seconds required to complete the task was measured.

(4) Object naming subtest of the Western Aphasia Battery (WAB) [19] for language.

(5) The word recall and word recognition subtests of the AD Assessment Scale (ADAS) [20] for episodic memory. The scores of true and false recall, true and false recognition, and $d^{\prime}$ were used, in which $d^{\prime}$ was calculated according to the formula adapted from the signal detection theory: $d^{\prime}=z$ (hit rate) $-z$ (false-alarm rate) [21]. In the formula, the hit and falsealarm rates were converted to $z$-scores by the $z$-transformation ( $z$ is the inverse of the standard normal distribution function).

(6) Visual discrimination (length and size, direction and complex form), overlapping figures, and visual counting tasks [22] for visuoperceptual and visuospatial functions.

Length and Size Discrimination. The stimuli consisted of 12 sheets of A4-sized paper, on each of which 2 or 3 lines, circles or rectangles were printed. Subjects were asked to indicate the longest and shortest, and the largest and smallest shapes. The total score ranged from 0 to 20 .

Direction Discrimination. Pairs of lines printed on a sheet of paper were presented to the subjects. Of the 15 pairs, 5 were parallel to each other, and the other 10 were inclined at angles from 4 to $7^{\circ}$. Subjects were asked to determine whether each pair of lines was parallel or not. Total score ranged from 0 to 15 .

Complex Form Discrimination. Four line-drawn geometric figures were placed in a $2 \times 2$ array on each of 20 sheets of paper. Of each set of 4 figures, 3 were the same and 1 was slightly different, rotated or flipped. Subjects were instructed to point to the odd figure. The maximum possible score was 20.

Overlapping Figures. Three sets of overlapping line drawings were used. Each set contained 3 simple geometric figures, 4 man-made objects or 5 fruits (a total of 12 objects). The subjects were asked to identify all individual figures by naming, describing, tracing by finger or matching them with non-overlapping drawings. The maximum possible score was 12 .

Visual Counting. There were 4-12 simple colored geometric figures (circles and triangles; red and blue) on each of 28 sheets of papers. Subjects were asked to count the number of figures with a specified color (red or blue) and form (circle or triangle) and the total number of figures. The maximum possible score was 56.

\section{Statistical Analyses}

Group comparisons on individual neuropsychological tests among the iNPH, AD and NC groups were made using the Kruskal-Wallis test. Post hoc pairwise comparisons were tested using the Mann-Whitney U test with Dunn-Sidak correction.

As a measure of each cognitive domain, a composite domain score on each of the $4 \mathrm{cog}$ nitive domains: executive function/attention $=$ digit span + spatial span + word fluency (phoneme + category) + TMT-A + FAB; episodic memory $=$ ADAS true word recall $+d^{\prime}$ of word recognition; language $=$ WAB object naming, and visuoperceptual/visuospatial function $=$ visual discrimination (direction and complex form) + overlapping figures + visual counting, was calculated. Each score was normalized by the following formula, which represents an alternative nonparametric procedure for parametric $z$-transformation: normalized score $=1-$ (median score of the patients/median score of the controls). Finally, we calculated the relative proportions (RP) of impairment in each cognitive domain to whole cognitive impairment according to the following formula:

$$
\mathrm{RP}(\%)=100 \times \frac{\text { mean normalized score of the domain }}{\text { sum of mean normalized scores across the } 4 \text { domains }}
$$

Comparisons of the scores before and after CSF shunt surgery were performed using the Wilcoxon test. 
Table 2. Neuropsychological test scores and time needed for the TMT-A in iNPH, AD and NC groups (means $\pm \mathrm{SD}$ )

\begin{tabular}{|c|c|c|c|c|c|c|}
\hline Test/subtest & $\begin{array}{l}\text { Total } \\
\text { score }\end{array}$ & $\begin{array}{l}\mathrm{iNPH} \\
(\mathrm{n}=32)\end{array}$ & $\begin{array}{l}\mathrm{AD} \\
(\mathrm{n}=32)\end{array}$ & $\begin{array}{l}\mathrm{NC} \\
(\mathrm{n}=30)\end{array}$ & $\mathrm{H}$ & $\mathrm{p}$ value \\
\hline MMSE & 30 & $21.6 \pm 4.6^{\mathrm{a}}$ & $21.3 \pm 3.4^{\mathrm{a}}$ & $28.7 \pm 1.3$ & 52.942 & $<0.001$ \\
\hline Digit span & & $7.7 \pm 1.8^{\mathrm{a}}$ & $8.5 \pm 1.3$ & $9.4 \pm 1.6$ & 13.543 & $<0.001$ \\
\hline Spatial span & & $7.6 \pm 1.9^{\mathrm{a}, \mathrm{b}}$ & $8.8 \pm 1.4^{\mathrm{a}}$ & $10.6 \pm 1.8$ & 32.172 & $<0.001$ \\
\hline \multicolumn{7}{|l|}{ Word fluency } \\
\hline Phoneme & & $11.5 \pm 6.2^{\mathrm{a}, \mathrm{b}}$ & $17.1 \pm 8.1$ & $22.1 \pm 7.8$ & 25.815 & $<0.001$ \\
\hline Category & & $7.1 \pm 4.1^{\mathrm{a}}$ & $8.2 \pm 3.0^{\mathrm{a}}$ & $15.1 \pm 5.3$ & 39.575 & $<0.001$ \\
\hline TMT-A, s & & $163.8 \pm 110.4^{\mathrm{a}, \mathrm{b}}$ & $96.9 \pm 65.4^{\mathrm{a}}$ & $52.3 \pm 19.5$ & 32.321 & $<0.001$ \\
\hline FAB & 18 & $9.9 \pm 3.0^{\mathrm{a}, \mathrm{b}}$ & $12.1 \pm 2.5^{\mathrm{a}}$ & $15.7 \pm 1.7$ & 47.928 & $<0.001$ \\
\hline WAB object naming & 60 & $57.8 \pm 3.2$ & $57.0 \pm 3.6$ & $58.7 \pm 1.6$ & 4.588 & 0.101 \\
\hline \multicolumn{7}{|l|}{ ADAS word recall } \\
\hline True recall & 30 & $12.9 \pm 4.0^{\mathrm{a}}$ & $13.7 \pm 3.5^{\mathrm{a}}$ & $21.5 \pm 2.8$ & 52.220 & $<0.001$ \\
\hline False recall & & $0.6 \pm 0.9$ & $0.9 \pm 1.6$ & $0.3 \pm 0.5$ & 2.973 & 0.226 \\
\hline \multicolumn{7}{|l|}{ ADAS word recognition } \\
\hline True recognition & 36 & $23.4 \pm 9.2^{\mathrm{a}}$ & $24.6 \pm 10.6^{\mathrm{a}}$ & $31.3 \pm 3.9$ & 15.869 & $<0.001$ \\
\hline False recognition & 36 & $0.7 \pm 2.4$ & $4.3 \pm 6.4^{\mathrm{a}, \mathrm{c}}$ & $0.1 \pm 0.3$ & 28.960 & $<0.001$ \\
\hline $\mathrm{d}^{\prime}$ & & $2.50 \pm 0.90^{\mathrm{a}}$ & $2.06 \pm 0.89^{\mathrm{a}}$ & $3.37 \pm 0.52$ & 33.245 & $<0.001$ \\
\hline \multicolumn{7}{|l|}{ Visual discrimination } \\
\hline Length and size & 20 & $20.0 \pm 0.0$ & $20.0 \pm 0.0$ & $20.0 \pm 0.0$ & 0.000 & 1.000 \\
\hline Direction & 15 & $13.8 \pm 2.3^{\mathrm{a}}$ & $13.7 \pm 1.8^{\mathrm{a}}$ & $14.8 \pm 0.5$ & 14.792 & $<0.001$ \\
\hline Complex form & 20 & $18.3 \pm 2.5^{\mathrm{a}, \mathrm{b}}$ & $19.6 \pm 0.7$ & $19.7 \pm 0.6$ & 14.620 & $<0.001$ \\
\hline Overlapping figures & 12 & $11.5 \pm 0.8^{\mathrm{a}}$ & $11.7 \pm 0.6$ & $12.0 \pm 0.2$ & 9.474 & $<0.001$ \\
\hline Visual counting & 56 & $49.9 \pm 5.3^{\mathrm{a}, \mathrm{b}}$ & $53.8 \pm 2.8$ & $55.2 \pm 1.0$ & 30.922 & $<0.001$ \\
\hline
\end{tabular}

$\mathrm{p}$ values are based on the Kruskal-Wallis test and the post hoc Mann-Whitney U test with Dunn-Sidak correction. Total scores indicate the maximum possible score. ${ }^{\mathrm{a}} \mathrm{p}<0.05$ vs. NC; ${ }^{\mathrm{b}} \mathrm{p}<0.05$ vs. AD; ${ }^{\mathrm{c}} \mathrm{p}<0.05$ vs. iNPH.

\section{Results}

The results are summarized in table 2 . There were significant differences among the three groups for all test scores $(\mathrm{p}<0.05)$ except for the WAB object naming $(\mathrm{p}=0.101)$, ADAS false recall $(\mathrm{p}=0.226)$, and length and size discrimination $(\mathrm{p}=1.000)$. The results of pairwise comparisons on the tests, in which significant group level differences were found, are described in detail below.

Although the iNPH group performed significantly worse than the NC group on the MMSE, there was no significant difference between the iNPH and AD groups. Compared to the NC group, the iNPH group performed significantly worse on the digit span and category fluency. There were no significant differences between the iNPH and AD groups on these tests. On the spatial span, phoneme fluency, TMT-A and FAB, the performance of the iNPH group was worse than the performances of the other two groups.

Compared to the NC group, the iNPH group was impaired on true recall, true recognition and $d^{\prime}$ of the ADAS, whereas the iNPH and AD groups were comparable on these measures. The iNPH patients made fewer false recognition responses than the AD patients. No significant differences were found between the iNPH and NC groups in terms of the number of false recognition responses.

Although the performance of the iNPH group was significantly worse than that of the $\mathrm{NC}$ group on the direction discrimination and overlapping figure, there was no significant 


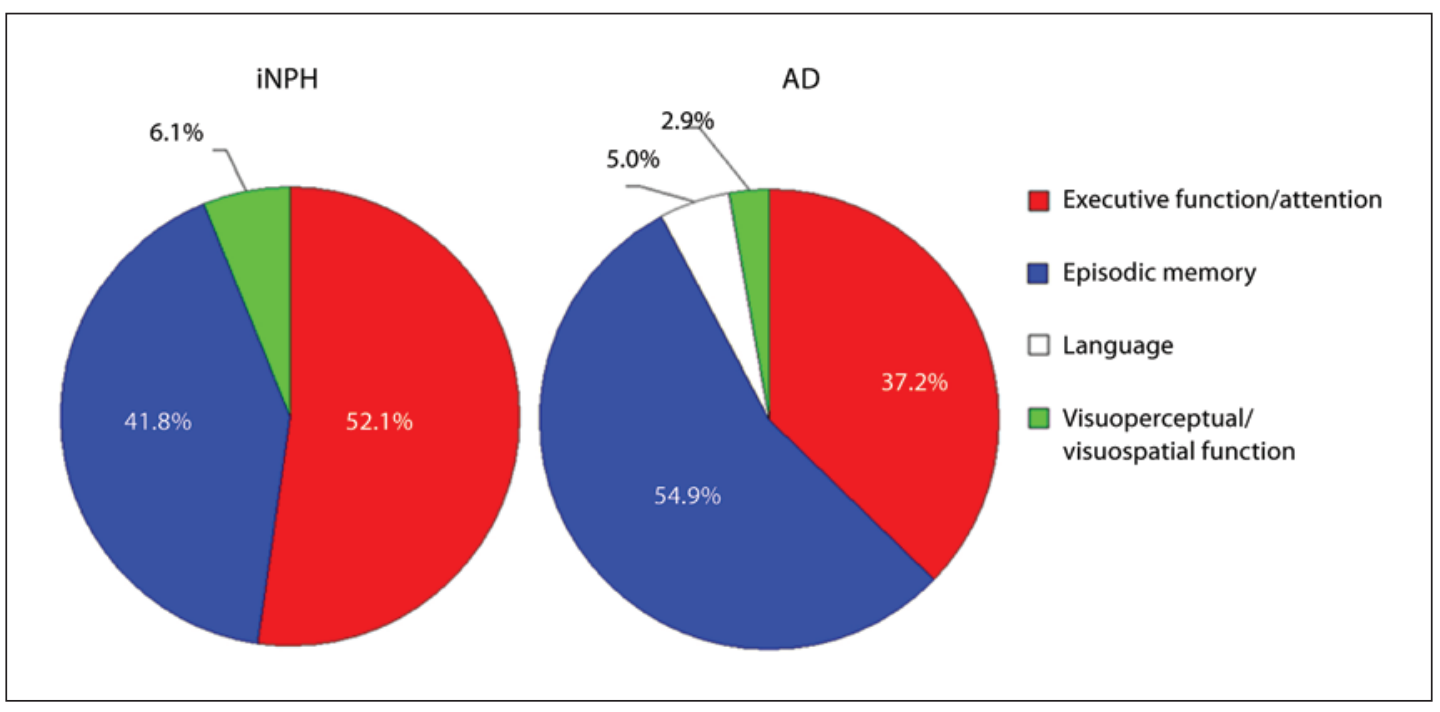

Fig. 1. Relative proportions of impairment in individual cognitive domains. Executive function/attention = digit span + spatial span + word fluency (phoneme + category) + TMT-A + FAB; episodic memory = ADAS true word recall $+d^{\prime}$ of word recognition; language $=$ WAB object naming; visuoperceptual/visuospatial function $=$ visual discrimination $($ direction + complex form $)+$ overlapping figures + visual counting .

difference between the iNPH and AD groups. On the complex form discrimination and visual counting, the performance was significantly worse in the iNPH group than the other two groups.

The relative proportion of impairment in each cognitive domain to whole cognitive impairment is illustrated in figure 1. Cognitive impairment in iNPH was dominated by executive/attentional deficits (52.1\% of the overall cognitive deficits). In the AD group, episodic memory impairment accounted for more than half of the overall cognitive deficits (54.9\%).

\section{Changes in Neuropsychological Test Performances after Shunt Surgery}

These results are summarized in table 3. One year after the CSF shunt surgery, performances of the TMT-A and FAB were significantly improved. A trend toward improvement was also observed in the visual counting score. The other 1-year test scores did not differ significantly versus before shunt surgery.

\section{Discussion}

Cognitive dysfunction in iNPH has long been classified under the category subcortical dementia, wherein the frontal dysexecutive syndrome is a dominant clinical manifestation. However, neuropsychology of iNPH has not been investigated in a comprehensive manner. A detailed characterization of cognitive dysfunction is essential for the differential diagnosis of preoperative iNPH and other dementia-type disorders. The present investigation revealed that patients with iNPH were impaired in broader cognitive domains than previously believed. These deficits extend beyond executive function, attention, and episodic memory to visuoperceptual and visuospatial functions. Although there were quantitative/statistical differences between iNPH and AD, including the more severe deficits in attention, executive function, and visuoperceptual and visuospatial functions that were observed in iNPH patients, it might be difficult to differentiate between the two disorders based on small sets of 
Table 3. Neuropsychological test scores and time needed for the TMT-A before and 1 year after shunt surgery (means $\pm \mathrm{SD})$

\begin{tabular}{lcccc}
\hline Test/subtest & $\begin{array}{l}\text { Total } \\
\text { score }\end{array}$ & $\begin{array}{l}\text { Before } \\
(\mathrm{n}=26)\end{array}$ & $\begin{array}{l}\text { After } \\
(\mathrm{n}=26)\end{array}$ & p value \\
\hline MMSE & 30 & $22.2 \pm 4.6$ & $23.2 \pm 4.5$ & 0.087 \\
Digit span & & $7.5 \pm 2.0$ & $7.7 \pm 1.8$ & 0.537 \\
Spatial span & $7.6 \pm 2.0$ & $7.7 \pm 1.6$ & 0.842 \\
Word fluency & & & \\
$\quad$ Phoneme & & $12.1 \pm 6.5$ & $12.3 \pm 6.5$ & 0.613 \\
$\quad$ Category & & $7.7 \pm 3.1$ & $7.8 \pm 3.9$ & 1.000 \\
TMT-A, s & & $165.1 \pm 119.0$ & $126.2 \pm 85.0$ & 0.027 \\
FAB & 18 & $10.3 \pm 2.9$ & $11.6 \pm 3.6$ & 0.013 \\
WAB object naming & 60 & $58.3 \pm 3.1$ & $58.8 \pm 2.4$ & 0.245 \\
ADAS word recall & & & & \\
$\quad$ True recall & 30 & $13.4 \pm 4.0$ & $13.8 \pm 5.0$ & 0.452 \\
$\quad$ False recall & & $0.5 \pm 0.9$ & $0.5 \pm 0.8$ & 0.771 \\
ADAS word recognition & & & & \\
$\quad$ True recognition & 36 & $23.9 \pm 8.2$ & $23.3 \pm 10.1$ & 0.788 \\
$\quad$ False recognition & 36 & $0.7 \pm 2.6$ & $0.2 \pm 0.5$ & 0.410 \\
$\quad d^{\prime}$ & & $2.56 \pm 0.80$ & $2.59 \pm 0.99$ & 0.798 \\
Visual discrimination & & & & \\
$\quad$ Length and size & 20 & $20.0 \pm 0.0$ & $20.0 \pm 0.0$ & 1.000 \\
$\quad$ Direction & 15 & $13.5 \pm 1.5$ & $13.5 \pm 1.6$ & 0.775 \\
$\quad$ Complex form & 20 & $18.3 \pm 2.3$ & $18.8 \pm 1.4$ & 0.138 \\
$\quad$ Overlapping figures & 12 & $11.5 \pm 0.8$ & $11.7 \pm 0.8$ & 0.350 \\
$\quad$ Visual counting & 56 & $50.6 \pm 5.4$ & $52.4 \pm 3.9$ & 0.074 \\
\hline
\end{tabular}

$\mathrm{p}$ values are based on the Wilcoxon test. Total scores indicate the maximum possible score.

neuropsychological tests on an individual patient basis because of the substantial overlap in each test score between iNPH and AD patients. We suggest that it is useful to identify the pattern of cognitive deficits of each disorder by examining broad cognitive domains. We found that frontal lobe dysfunctions accounted for $>50 \%$ of the whole cognitive deficit in iNPH patients, whereas memory impairment accounted for $>50 \%$ of the cognitive deficit in AD patients (fig. 1).

Consistent with previous studies, iNPH patients displayed impaired executive function and attention. This finding is consistent with the cognitive features of subcortical dementia, which arises from disruption of the frontal-subcortical circuits [23-25]. Previous neuroimaging studies have reported that frontal cortical grey matter volume is preserved, but frontal regional cerebral blood flow (rCBF) is decreased in iNPH patients [26-29]. These findings support the view that frontal lobe-like cognitive dysfunction in iNPH is associated with subcortical white matter damage but not frontal lobe damage itself.

It has been proposed that the pattern of memory deficit in iNPH is of a 'frontal lobe' type, in which recall is disproportionately affected relative to recognition memory $[10,11]$. In contrast, $\mathrm{AD}$ patients are reportedly impaired in recall as well as in recognition [30]. However, we found that both recognition and recall were impaired in a similar fashion in the $\mathrm{iNPH}$ and AD groups, suggesting that memory impairment in iNPH is not exclusively ascribable to frontal lobe dysfunction. A recent neuroimaging study demonstrated a reduction in the medial temporal volume in iNPH [28]. We suggest that the medial temporal lobe damage may be associated with memory impairment in iNPH [28]. Increased false recognition in $\mathrm{AD}$ was the only feature in the memory domain that differentiated between the two dis- 
orders [31]. Some investigators have stressed the significance of executive dysfunction in the mechanism of false recognition [32]. However, the lower rate of false recognition in iNPH patients is inexplicable by this hypothesis, because the impairment in executive function was higher in iNPH than in AD patients. Consistent with the present findings, previous studies have demonstrated that patients with depressive pseudo-dementia [33] and progressive supranuclear palsy [34] made fewer false recognition errors than those with $\mathrm{AD}$. Conservative response bias due to apathy or psychomotor slowing may be associated with less false recognition in patients with iNPH or other subcortical dementias [35].

Visuoperceptual and visuospatial functions have not been addressed in previous studies of iNPH, and the present investigation demonstrated significant impairment in these functions. In particular, the impairment in the visual discrimination and the visual counting tasks was more severe in iNPH than in AD patients. Defective performance on the visual discrimination tasks suggests impairment in visual form perception or constructive function [22]. Previously, a constructive deficit in iNPH has been demonstrated using the block design task of the WAIS-R [12]. Although the visual counting task requires working memory, patients with frontal lobe damage performed normally on this task in a previous study. This suggests the primary contribution of visuospatial function, which is subserved by the parietal cortex, in this task [36]. These findings are consistent with previous neuroimaging studies that showed a parietal rCBF reduction in iNPH $[13,29]$.

Our longitudinal analysis revealed that CSF shunt surgery improved TMT-A and FAB performance. Although not statistically significant, we also found a trend towards an improvement in the visual counting task. A previous study in healthy subjects reported a $3.6 \%$ improvement in the test-retest of the TMT-A with a 1-year interval [37], whereas the present study demonstrated a $23.5 \%$ improvement 1 year after surgery compared to the baseline values. Although there is no published report on the practice effects in FAB and the visual counting task, our preliminary investigation of 20 patients with AD showed no practice effects in the 1-week-interval test-retest of FAB [38]. These findings suggest that the longitudinal improvement observed in the present study is not ascribable to a practice effect. This interpretation is supported by previous neuroimaging studies in which frontal and parietal rCBF was improved after shunt surgery $[39,40]$.

Lastly, a major limitation of the study should be noted. Because of the lack of pathological confirmation, we are unable to rule out the possibility that some of the present iNPH patients might have had AD pathology. Concomitant AD pathology would have affected baseline neuropsycholgical performance and postoperative improvements [41].

\section{Acknowledgments}

This study was supported by the Global COE Program (Basic \& Transitional Research Center for Global Brain Science), MEXT, Japan, and Health and Labor Sciences Research Grants for Research on Intractable Diseases, Ministry of Health, Labor and Welfare.

\section{Disclosure Statement}

The authors declare no conflicts of interest. 


\section{References}

1 Hebb AO, Cusimano MD: Idiopathic normal pressure hydrocephalus: a systematic review of diagnosis and outcome. Neurosurgery 2001;49:1166-1184.

-2 Brean A, Eide PK: Prevalence of probable idiopathic normal pressure hydrocephalus in a Norwegian population. Acta Neurol Scand 2008;118:48-53.

-3 Hiraoka K, Meguro K, Mori E: Prevalence of idiopathic normal-pressure hydrocephalus in the elderly population of a Japanese rural community. Neurol Med Chir (Tokyo) 2008:48:197-199.

-4 Hakim S, Adams RD: The special clinical problem of symptomatic hydrocephalus with normal cerebrospinal fluid pressure. Observations on cerebrospinal fluid hydrodynamics. J Neurol Sci 1965;2: 307-327.

-5 Adams RD, Fisher CM, Hakim S, Ojemann RG, Sweet WH: Symptomatic occult hydrocephalus with 'normal' cerebrospinal-fluid pressure. A treatable syndrome. N Engl J Med 1965;273:117-126.

-6 Ishikawa M, Hashimoto M, Kuwana N, Mori E, Miyake H, Wachi A, Takeuchi T, Kazui H, Koyama H: Guidelines for management of idiopathic normal pressure hydrocephalus. Neurol Med Chir (Tokyo) 2008;48(suppl):S1-S23.

7 Marmarou A, Bergsneider M, Relkin N, Klinge P, Black PM: Development of guidelines for idiopathic normal-pressure hydrocephalus: introduction. Neurosurgery 2005;57:1-3.

-8 Iseki C, Kawanami T, Nagasawa H, Wada M, Koyama S, Kikuchi K, Arawaka S, Kurita K, Daimon M, Mori E, Kato T: Asymptomatic ventriculomegaly with features of idiopathic normal pressure hydrocephalus on MRI (AVIM) in the elderly: a prospective study in a Japanese population. J Neurol Sci 2009;277:54-57.

-9 Hashimoto M, Ishikawa M, Mori E, Kuwana N, The Study of INPH on Neurological Improvement (SINPHONI): Diagnosis of idiopathic normal pressure hydrocephalus is supported by MRI-based scheme: a prospective cohort study. Cerebrospinal Fluid Res 2010;7:18.

10 Iddon JL, Pickard JD, Cross JJ, Griffiths PD, Czosnyka M, Sahakian BJ: Specific patterns of cognitive impairment in patients with idiopathic normal pressure hydrocephalus and Alzheimer's disease: a pilot study. J Neurol Neurosurg Psychiatry 1999;67:723-732.

-11 Walchenbach R, Geiger E, Thomeer RT, Vanneste JA: The value of temporary external lumbar CSF drainage in predicting the outcome of shunting on normal pressure hydrocephalus. J Neurol Neurosurg Psychiatry 2002;72:503-506.

-12 Ogino A, Kazui H, Miyoshi N, Hashimoto M, Ohkawa S, Tokunaga H, Ikejiri Y, Takeda M: Cognitive impairment in patients with idiopathic normal pressure hydrocephalus. Dement Geriatr Cogn Disord 2006;21:113-119.

-13 Sasaki H, Ishii K, Kono AK, Miyamoto N, Fukuda T, Shimada K, Ohkawa S, Kawaguchi T, Mori E: Cerebral perfusion pattern of idiopathic normal pressure hydrocephalus studied by SPECT and statistical brain mapping. Ann Nucl Med 2007;21:39-45.

-14 Kubo Y, Kazui H, Yoshida T, Kito Y, Kimura N, Tokunaga H, Ogino A, Miyake H, Ishikawa M, Takeda M: Validation of grading scale for evaluating symptoms of idiopathic normal-pressure hydrocephalus. Dement Geriatr Cogn Disord 2008;25:37-45.

- 15 McKhann G, Drachman D, Folstein M, Katzman R, Price D, Stadlan EM: Clinical diagnosis of Alzheimer's disease: report of the NINCDS-ADRDA Work Group under the auspices of Department of Health and Human Services Task Force on Alzheimer's Disease. Neurology 1984;34:939-944.

16 Mori E, Mitani Y, Yamadori A: Usefulness of Japanese version of the Mini-Mental State Examination in neurological patients (in Japanese). Jpn J Neuropsychol 1985;1:82-90.

17 Abe M, Suzuki K, Okada K, Miura R, Fujii T, Mori E, Yamadori A: Normative data on tests for frontal lobe functions: Trail Making Test, Verbal Fluency, Wisconsin Card Sorting Test (Keio version) (in Japanese). No To Shinkei 2004;56:567-574.

-18 Dubois B, Slachevsky A, Litvan I, Pillon B: The FAB: a frontal assessment battery at bedside. Neurology 2000;55:1621-1626.

19 Sugishita M: The Western Aphasia Battery (Japanese edition). Tokyo, Igakushoin, 1986.

20 Homma A, Fukuzawa K, Tsukada Y, Ishii T, Hasegawa K, Mohs R: Development of a Japanese version of the Alzheimer's disease assessment scale (ADAS). Jpn J Geriatr Psychiatry 1992;3:647-655.

-21 Snodgrass JG, Corwin J: Pragmatics of measuring recognition memory: applications to dementia and amnesia. J Exp Psychol Gen 1988;117:34-50. 
-22 Fujimori M, Imamura T, Hirono N, Ishii K, Sasaki M, Mori E: Disturbances of spatial vision and object vision correlate differently with regional cerebral glucose metabolism in Alzheimer's disease. Neuropsychologia 2000;38:1356-1361.

-23 Gallassi R, Morreale A, Montagna P, Sacquegna T, Di Sarro R, Lugaresi E: Binswanger's disease and normal-pressure hydrocephalus. Clinical and neuropsychological comparison. Arch Neurol 1991;48: 1156-1159.

-24 Vanneste JA: Diagnosis and management of normal-pressure hydrocephalus. J Neurol 2000;247: 5-14.

-25 Cummings JL: Subcortical dementia. Neuropsychology, neuropsychiatry, and pathophysiology. Br J Psychiatry 1986;149:682-697.

-26 Murakami M, Hirata Y, Kuratsu JI: Predictive assessment of shunt effectiveness in patients with idiopathic normal pressure hydrocephalus by determining regional cerebral blood flow on 3D stereotactic surface projections. Acta Neurochir (Wien) 2007;149:991-997.

-27 Kristensen B, Malm J, Fagerlund M, Hietala SO, Johansson B, Ekstedt J, Karlsson T: Regional cerebral blood flow, white matter abnormalities, and cerebrospinal fluid hydrodynamics in patients with idiopathic adult hydrocephalus syndrome. J Neurol Neurosurg Psychiatry 1996;60:282-288.

-28 Ishii K, Kawaguchi T, Shimada K, Ohkawa S, Miyamoto N, Kanda T, Uemura T, Yoshikawa T, Mori E: Voxel-based analysis of gray matter and CSF space in idiopathic normal pressure hydrocephalus. Dement Geriatr Cogn Disord 2008;25:329-335.

-29 Takaya M, Kazui H, Tokunaga H, Yoshida T, Kito Y, Wada T, Nomura K, Shimosegawa E, Hatazawa J, Takeda M: Global cerebral hypoperfusion in preclinical stage of idiopathic normal pressure hydrocephalus. J Neurol Sci 2010;298:35-41.

-30 Moss MB, Albert MS, Butters N, Payne M: Differential patterns of memory loss among patients with Alzheimer's disease, Huntington's disease, and alcoholic Korsakoff's syndrome. Arch Neurol 1986; 43:239-246.

-31 Hildebrandt H, Haldenwanger A, Eling P: False recognition helps to distinguish patients with Alzheimer's disease and amnestic MCI from patients with other kinds of dementia. Dement Geriatr Cogn Disord 2009;28:159-167.

-32 Budson AE, Sullivan AL, Mayer E, Daffner KR, Black PM, Schacter DL: Suppression of false recognition in Alzheimer's disease and in patients with frontal lobe lesions. Brain 2002;125:2750-2765.

-33 Gainotti G, Marra C: Some aspects of memory disorders clearly distinguish dementia of the Alzheimer's type from depressive pseudo-dementia. J Clin Exp Neuropsychol 1994;16:65-78.

-34 Gainotti G, Marra C, Villa G, Parlato V, Chiarotti F: Sensitivity and specificity of some neuropsychological markers of Alzheimer dementia. Alzheimer Dis Assoc Disord 1998;12:152-162.

-35 Lindqvist G, Andersson H, Bilting M, Blomstrand C, Malmgren H, Wikkelso C: Normal pressure hydrocephalus: psychiatric findings before and after shunt operation classified in a new diagnostic system for organic psychiatry. Acta Psychiatr Scand Suppl 1993;373:18-32.

- 36 Clague F, Dudas RB, Thompson SA, Graham KS, Hodges JR: Multidimensional measures of person knowledge and spatial associative learning: can these be applied to the differentiation of Alzheimer's disease from frontotemporal and vascular dementia? Neuropsychologia 2005;43:1338-1350.

-37 Solana E, Poca MA, Sahuquillo J, Benejam B, Junqué C, Dronavalli M: Cognitive and motor improvement after retesting in normal-pressure hydrocephalus: a real change or merely a learning effect? J Neurosurg 2010;112:399-409.

- 38 Saito M, Nishio Y, Kanno S, Mori E: Discriminating idiopathic normal pressure hydrocephalus from Alzheimer's disease: distinctive cognitive profiles and the contribution of the CSF tap test (abstract). Eur J Neurol 2008;15:299.

-39 Larsson A, Bergh AC, Bilting M, Arlig A, Jacobsson L, Stephensen H, Wikkelso C: Regional cerebral blood flow in normal pressure hydrocephalus: diagnostic and prognostic aspects. Eur J Nucl Med 1994;21:118-123.

-40 Mataro M, Poca MA, Salgado-Pineda P, Castell-Conesa J, Sahuquillo J, Diez-Castro MJ, AguadeBruix S, Vendrell P, del Mar Matarin M, Junque C: Postsurgical cerebral perfusion changes in idiopathic normal pressure hydrocephalus: a statistical parametric mapping study of SPECT images. J Nucl Med 2003;44:1884-1889.

-41 Hamilton R, Patel S, Lee EB, Jackson EM, Lopinto J, Arnold SE, Clark CM, Basil A, Shaw LM, Xie SX, Grady MS, Trojanowski JQ: Lack of shunt response in suspected idiopathic normal pressure hydrocephalus with Alzheimer disease pathology. Ann Neurol 2010;68:535-540. 